\title{
Integrable dynamics of coupled Fermi-Bose condensates
}

\author{
Emil A. Yuzbashyan ${ }^{1}$, Vadim B. Kuznetsov ${ }^{2}$, and Boris L. Altshuler ${ }^{3}$ \\ ${ }^{1}$ Center for Materials Theory, Department of Physics and Astronomy, \\ Rutgers University, Piscataway, New Jersey 08854, USA \\ ${ }^{2}$ Department of Applied Mathematics, University of Leeds, Leeds, LS2 9JT, UK \\ ${ }^{3}$ Physics Department, Princeton University, Princeton, NJ 08544, USA
}

\begin{abstract}
We study the mean-field dynamics of a fermionic condensate interacting with a single bosonic mode (a generalized Dicke model). This problem is integrable and can be mapped onto a corresponding BCS problem. We derive the general solution and a full set of integrals of motion for the time evolution of coupled Fermi-Bose condensates. The present paper complements our earlier study of the dynamics of the BCS model (cond-mat/0407501 and cond-mat/0505493). Here we provide a self-contained introduction to the variable separation method, which enables a complete analytical description of the evolution of the generalized Dicke, BCS, and other similar models.
\end{abstract}




\section{INTRODUCTION}

A number of closely related non-stationary problems have come up recently in different contexts. In these problems the goal is to describe the dynamics of a many-body system following a sudden perturbation that drove the system out of an equilibrium. The system in question can be a BCS superconductor 1, 2, 3, 4, 5, 6, 17, 8, 9, 10, 11], coupled FermiBose condensates 12, 13], or a single electronic spin interacting with many nuclear spins (the central spin model) 14, 15, 16, 17, 18, 19, 20]. A common feature of all these problems is that they can be formulated in terms of spin Hamiltonians, which belong to a class of integrable systems known as Gaudin magnets [21, 22, 23]. It turns out that this fact enables one to solve for the evolution of all these systems using the same approach.

In the present paper we focus on a particular example of such a problem. Namely, we study the dynamics of a fermionic condensate interacting with a single bosonic mode. Recent studies of this problem [12, 13] were motivated by experiments 24, 25, 26, 27] on cold fermion pairing. The idea was that near the Feshbach resonance [28, 29] these systems can be modeled by a fermionic condensate of atoms strongly coupled to the lowest energy bosonic mode.

We assume that the system has been prepared in a nonequilibrium state at $t \leq 0$ and study the subsequent evolution for $t>0$. Our main result is a complete solution for the dynamics of the system. We also derive a full set of its integrals of motion. It turns out that the mean-field time evolution of the coupled Fermi-Bose condensates can be mapped onto the corresponding BCS problem, which was solved previously in Refs. 9, 10. For this reason, the emphasis in the present paper is on this mapping and detailed or alternative derivations that have been largely omitted in Refs. 9 , 10.

The fermion-boson condensate is described by the following Hamiltonian:

$$
\hat{H}=\sum_{j, \sigma} \epsilon_{j} \hat{c}_{j \sigma}^{\dagger} \hat{c}_{j \sigma}+\omega \hat{b}^{\dagger} \hat{b}+g \sum_{j}\left(\hat{b}^{\dagger} \hat{c}_{j \downarrow} \hat{c}_{j \uparrow}+\hat{b} \hat{c}_{j \uparrow}^{\dagger} \hat{c}_{j \downarrow}^{\dagger}\right)
$$

where $\epsilon_{j}$ are the single-particle energy levels and the operators $\hat{c}_{j \sigma}^{\dagger}\left(\hat{c}_{j \sigma}\right)$ create (annihilate) a fermion of one of the two species $\sigma=\uparrow$ or $\downarrow$ in an orbital eigenstate of energy $\epsilon_{j}$. Eigenstates $|j \uparrow\rangle$ and $|j \downarrow\rangle$ are related by the time-reversal symmetry [30]. For example, if the singleparticle potential is translationally invariant, $|j \uparrow\rangle=|\mathbf{p} \uparrow\rangle$ and $|j \downarrow\rangle=|-\mathbf{p} \downarrow\rangle$. Operators $\hat{b}^{\dagger}(\hat{b})$ create (annihilate) quanta of the bosonic field. 
We study the dynamics of the fermion-oscillator model (1.1) in the mean-field approximation. This amounts to treating the bosonic field classically, i.e. replacing operators $\hat{b}^{\dagger}$ and $\hat{b}$ with $c$-numbers in the Heisenberg equations of motion for Hamiltonian (1.1). This procedure is expected to be exact as long as the bosonic mode is macroscopically populated. It turns out that in this approximation the dynamics coincides with that of a classical Hamiltonian system, which can be mapped onto the corresponding BCS problem (see below). The classical dynamical variables are the time-dependent quantum-mechanical expectation values $\left\langle\hat{c}_{j \downarrow} \hat{c}_{j \uparrow}\right\rangle,\langle\hat{b}\rangle$, and $\left\langle\sum_{\sigma} \hat{c}_{j \sigma}^{\dagger} \hat{c}_{j \sigma}\right\rangle$. We will see below that the mean-field approximation is also equivalent to the usual way of obtaining the classical limit - by replacing operators with classical variables and their commutators with Poisson brackets.

Here we assume that the number of energy levels, $n$, interacting with the bosonic field in the system (1.1) is arbitrary large, but finite. In this case the typical evolution is quasi-periodic with $n+1$ incommensurate frequencies. The system exhibits irregular multifrequency oscillations ergodically exploring the part of the phase-space allowed by the conservation laws, returning arbitrarily close to its initial state at irregular time intervals. For finite $n$, the solution, albeit explicit, is rather complicated. However, it considerably simplifies in the thermodynamic limit $n \rightarrow \infty$. In this limit, the return time diverges for most physical initial conditions, while the solution decays to a simple limiting dynamics at large times 31]. For example, following an initial decay, $|\langle\hat{b}(t)\rangle|$ asymptotes either to a constant value (cf. Refs. 2, 3, 7, 11) or to an oscillatory behavior characterized by only a few frequencies (cf. Fig. 2 in Refs. 4, 6 ).

The fermion-oscillator model (1.1) can be viewed as a generalization of the Dicke (TavisCummings) model of Quantum Optics. The latter can be obtained from (1.1) in the zero fermionic bandwidth limit, i.e. when all single-particle levels $\epsilon_{j}$ are degenerate, $\epsilon_{j}=\mu$. To see this, it is useful to reformulate the model (1.1) as a spin-oscillator model using Anderson's pseudospin representation[1]. Pseudospins are defined as

$$
2 \hat{K}_{j}^{z}=\hat{n}_{j}-1, \quad \hat{K}_{j}^{-}=\hat{c}_{j \downarrow} \hat{c}_{j \uparrow}, \quad \hat{K}_{j}^{+}=\hat{c}_{j \uparrow}^{\dagger} \hat{c}_{j \downarrow}^{\dagger},
$$

where $\hat{n}_{j}=\sum_{\sigma} \hat{c}_{j \sigma}^{\dagger} \hat{c}_{j \sigma}$. Operators $\hat{\mathbf{K}}_{j}$ have all properties of spin-1/2 on the subspace of unoccupied and doubly occupied (unblocked) levels $\epsilon_{j}$. Singly occupied (blocked) levels do not interact with the bosonic field and are decoupled from the dynamics. In terms of 
pseudospins the Hamiltonian (1.1) for $n$ unblocked levels takes the form

$$
\hat{H}=\sum_{j=0}^{n-1} 2 \epsilon_{j} \hat{K}_{j}^{z}+\omega \hat{b}^{\dagger} \hat{b}+g \sum_{j=0}^{n-1}\left(\hat{b}^{\dagger} \hat{K}_{j}^{-}+\hat{b} \hat{K}_{j}^{+}\right) .
$$

In the zero bandwidth limit the Hamiltonian (1.3) describes an interaction of a single collective spin $\hat{\mathbf{T}}=\sum_{j} \hat{\mathbf{K}}_{j}$ with a harmonic oscillator

$$
\hat{H}_{\text {Dicke }}=2 \mu \hat{T}_{z}+\omega \hat{b}^{\dagger} \hat{b}+g\left(\hat{b}^{\dagger} \hat{T}_{-}+\hat{b} \hat{T}_{+}\right) .
$$

This model was suggested by R. H. Dicke in 1953 [32]. Its spectrum was obtained exactly by M. Tavis and F. W. Cummings in 1967 [33]. In the mean-field approximation it becomes a classical model for the dynamical variables $\langle\hat{\mathbf{T}}(t)\rangle$ and $\langle\hat{b}(t)\rangle$ with only two degrees of freedom (see also below). The mean-field solution for the time evolution was outlined by Dicke 32] and derived in detail by R. Bonifacio and G. Preparata in 1969 34]. In fact, the resulting classical problem is that of a spherical pendulum. The solution for $\langle\hat{\mathbf{T}}(t)\rangle$ and $\langle\hat{b}(t)\rangle$ is in terms of elliptic functions and (apart from the azimuthal motion of the pendulum) is simply periodic 34].

Remarkably, a more general many-body model (1.11.3) also turns out to be integrable. This was established by M. Gaudin in 1972 [21] and later by B. Jurco[35] who used a different approach. First explicit nonlinear solutions for the mean-field dynamics of the model (1.11.3) were constructed in Refs. 12, 13. Interestingly, the initial conditions for which these solutions occur are such that the dynamics of the model (1.111.3) reduces to that of the Dicke model (1.4), i.e. it can be described in terms of a single collective spin coupled to the bosonic field. Similar solutions for the mean-field dynamics of the BCS model were discovered by R. A. Barankov et. al. in Ref. 6 and in an unpublished work by V. S. Shumeiko [5].

Here we solve for the dynamics of the model (1.11.3) in the mean-field approximation for arbitrary initial conditions. We also derive a full set of conservation laws for the mean-field dynamics. The typical evolution of the system is quasi-periodic with $n+1$ incommensurate frequencies. However, for certain special choices of initial conditions the dynamics is characterized by $m+1<n+1$ incommensurate frequencies and can be described in terms of $m$ collective spins coupled to the bosonic field.

Our approach to the mean-field dynamics of the fermion-oscillator model (1.1) employs the method of separation of variables. This method was suggested by I. V. Komarov 37] and later developed by E. Sklyanin and V. Kuznetsov 22, 23]. It allows us to derive a full set of 
integrals of motion for both the quantum model (1.11.3) and its classical counterpart. We also use it to derive and analyze equations of motion in terms of separation variables.

Upon the replacement of the quantum bosonic field $\hat{b}$ with its time-dependent expectation value, the Hamiltonian (1.1) becomes similar to the mean-field BCS Hamiltonian. In this analogy, $g\langle\hat{b}(t)\rangle$ plays the role of the BCS gap $\Delta(t)$. The difference is that in the case of the BCS model $\Delta(t)$ is not an independent dynamical variable, but is related to $\left\langle\hat{c}_{j \downarrow}(t) \hat{c}_{j \uparrow}(t)\right\rangle=$ $\left\langle\hat{K}_{j}^{-}(t)\right\rangle$ by the self-consistency condition $\Delta(t)=g \sum_{j}\left\langle\hat{K}_{j}^{-}(t)\right\rangle$. Nevertheless, it turns out to be possible to map the mean-field dynamics of the fermion-oscillator model (1.1) with $n$ energy levels onto the corresponding BCS problem with $n+1$ levels. This mapping is facilitated by the variable separation and enables us to obtain the general solution for the time dependence of the expectation values $\left\langle\hat{\mathbf{K}}_{j}(t)\right\rangle$ and $\langle\hat{b}(t)\rangle$ in terms of hyperelliptic functions [38, 39] using the known solution for the mean-field dynamics of the BCS model 9 , 10].

The rest of the paper is organized as follows. In section II we derive the classical Hamiltonian that governs the mean-field dynamics of the quantum model (1.111.3). We show that the dynamics of the resulting classical model is integrable and derive a full set of its conservation laws in section III. In section IV we perform a transformation to a new set of variables, which facilitates the solution of the equations of motion. The mapping to the corresponding BCS problem and the general solution for the mean-field dynamics of the model (1.11.3) are derived in section $\nabla 1$. Finally, section $\nabla I T$ is devoted to an interesting class of particular solutions that include mean-field equilibrium states and special solutions of Refs. [12, 13, 32, 34].

\section{CLASSICAL MODEL}

Here we derive the classical Hamiltonian model that describes the mean-field dynamics of the quantum model (1.11.3).

We start with the Heisenberg equations of motion for the spin-oscillator model (1.3).

$$
\begin{array}{ll}
\dot{\hat{\mathbf{K}}}_{j}=i\left[\hat{H}, \hat{\mathbf{K}}_{j}\right]=\hat{\mathbf{B}}_{j} \times \hat{\mathbf{K}}_{j} & \hat{\mathbf{B}}_{j}=\left(2 g \hat{b}_{x}, 2 g \hat{b}_{y}, 2 \epsilon_{j}\right), \\
\dot{\hat{b}}=i[\hat{H}, \hat{b}]=-i \omega \hat{b}-i g \hat{T}_{-} \quad \hat{\mathbf{T}}=\sum_{q=0}^{n-1} \hat{\mathbf{K}}_{q},
\end{array}
$$


where the operators $\hat{b}_{x}$ and $\hat{b}_{y}$ are defined by

$$
\hat{b}=\hat{b}_{x}-i \hat{b}_{y} \quad \hat{b}^{\dagger}=\hat{b}_{x}+i \hat{b}_{y}
$$

In the regime when the bosonic mode is macroscopically populated, we can replace operators $\hat{b}(t)$ and $\hat{b}^{\dagger}(t)$ in Eqs. (2.1) with $c$-numbers: $b(t)=\langle\hat{b}(t)\rangle$ and $\bar{b}(t)=\left\langle\hat{b}^{\dagger}(t)\right\rangle$, where $\langle\ldots\rangle$ stands for the time-dependent quantum-mechanical expectation value. After this replacement, Eqs. (2.1) become linear in operators. Taking their quantum-mechanical expectation value, we obtain for $\mathbf{s}_{j}(t)=\left\langle\hat{\mathbf{K}}_{j}(t)\right\rangle$

$$
\begin{aligned}
& \dot{\mathbf{s}}_{j}=\mathbf{B}_{j} \times \mathbf{s}_{j} \quad \mathbf{B}_{j}=\left(2 g b_{x}, 2 g b_{y}, 2 \epsilon_{j}\right), \\
& \dot{b}=-i \omega b-i g J_{-} \quad \mathbf{J}=\sum_{j=0}^{n-1} \mathbf{s}_{j} .
\end{aligned}
$$

An important observation is that Eqs. (2.2) are Hamiltonian equations for the following classical model:

$$
H=\sum_{j=0}^{n-1} 2 \epsilon_{j} s_{j}^{z}+\omega \bar{b} b+g \sum_{j=0}^{n-1}\left(\bar{b} s_{j}^{-}+b s_{j}^{+}\right),
$$

where $s_{j}^{ \pm}=s_{j}^{x} \pm i s_{j}^{y}$. This Hamiltonian governs $n$ classical spins interacting with a classical harmonic oscillator [40].

Classical spins are similar to the angular momentum and have the same Poisson brackets

$$
\begin{aligned}
& \left\{s_{j}^{a}, s_{j}^{b}\right\}=-\varepsilon_{a b c} s_{j}^{c}, \\
& \{b, \bar{b}\}=i \quad\left\{b_{x}, b_{y}\right\}=\frac{1}{2},
\end{aligned}
$$

where $a, b$, and $c$ stand for the spatial indexes $x, y$, and $z$. All other Poisson brackets between components of $\mathbf{s}_{j}$ and $b$ vanish. Equations (2.4) determine the fundamental Poisson brackets in our problem in a sense that Poisson brackets between any other pair of dynamical variables (functions of $b, \bar{b}$ and components of $\mathbf{s}_{j}$ ) can be obtained from Eqs. (2.4) using the standard properties of Poisson brackets [41]. For example, Eqs. (2.2) can be obtained from the Hamiltonian equations of motion $\dot{\mathbf{s}}_{j}=\left\{H, \mathbf{s}_{j}\right\}$ and $\dot{b}=\{H, b\}$ using Eqs. (2.4) and (2.3).

Equations of motion (2.2) conserve the length of each spin, $\mathbf{s}_{j}^{2}=$ const. This fact can also be viewed as a property of the brackets (2.4) - since $\mathbf{s}_{j}^{2}$ Poisson-commutes with all other dynamical variables, its bracket with the Hamiltonian (2.3) also vanishes. In the mean-field 
approximation eigenstates of the Hamiltonian (1.3) are product states. In a pure state of a spin- $1 / 2$, there is always an axis, $\mathbf{n}$, such that the projection of the spin onto it is $1 / 2$, i.e. $\hat{\mathbf{K}}_{j} \cdot \mathbf{n}=1 / 2$. In this case, $\mathbf{s}_{j}^{2}=\left\langle\hat{\mathbf{K}}_{j}\right\rangle^{2}=1 / 4$. Therefore, if the system was in a product state at $t=0$

$$
\mathbf{s}_{j}^{2}=\left(s_{j}^{z}\right)^{2}+s_{j}^{+} s_{j}^{-}=\frac{1}{4} .
$$

If a number of orbitals $\epsilon_{j}$ in (2.3) are degenerate, the magnitude of their total classical spin $\sum_{\epsilon_{j}=\epsilon} \mathbf{s}_{j}$ is conserved by the equations of motion (2.2). In this case, one can replace the corresponding spins $\mathbf{s}_{j}$ with a single classical spin of a larger magnitude,

$$
\sum_{\epsilon_{j}=\epsilon} \epsilon_{j} \mathbf{s}_{j}=\epsilon \mathbf{S}_{\epsilon}, \quad \text { where } \quad \mathbf{S}_{\epsilon}=\sum_{\epsilon_{j}=\epsilon} \mathbf{s}_{j}
$$

in the Hamiltonian (2.3) and sum over nondegenerate orbitals only. Below we will assume whenever necessary that such a replacement has been made, i.e. that orbitals $\epsilon_{j}$ are nondegenerate.

Finally, comparing Hamiltonians (1.3) and (2.3) and brackets (2.4) to the corresponding quantum commutators, we note that the mean-field approximation is equivalent to the standard procedure of going from quantum to classical mechanics by replacing commutators with Poisson brackets, $i[A, B] \rightarrow\{A, B\}$ (here $\hbar=1$ ).

\section{INTEGRABILITY}

Here we demonstrate that the classical model (2.3), as well as its quantum counterparts (1.3) and (1.1), are integrable and introduce a useful tool to analyze and solve for their dynamics.

The Hamiltonian (2.3) depends on $2(n+1)$ dynamical variables: 2 angles for each of $n$ spins plus the coordinate and the momentum of the oscillator 40]. Therefore, its phase space is $2(n+1)$-dimensional and the number of the degrees of freedom (the number of generalized coordinates) is $n+1$. To show that the classical model (2.3) is integrable, we have to show that it has $n+1$ independent integrals of motion (see e.g. Refs. 36, 41). 
Consider the following vector-functions of an auxiliary parameter $u$ :

$$
\begin{aligned}
& \mathbf{L}_{j}(u)=\frac{\mathbf{s}_{j}}{u-\epsilon_{j}} \quad j=0, \ldots, n-1 \\
& \mathbf{L}_{n}(u)=\frac{1}{g^{2}}\left(\begin{array}{c}
2 g b_{x} \\
2 g b_{y} \\
2 u-\omega
\end{array}\right) .
\end{aligned}
$$

It follows from the above definitions and Eqs. (2.4) that components of $\mathbf{L}_{k}(u)$ have the following Poisson brackets:

$$
\left\{L_{k}^{a}(v), L_{k}^{b}(w)\right\}=\frac{\varepsilon_{a b c}}{v-w}\left(L_{k}^{c}(v)-L_{k}^{c}(w)\right) \quad k=0, \ldots, n
$$

Because components of different $\mathbf{L}_{j}(u)$ Poisson-commute with each other, their sum

$$
\mathbf{L}(u)=\frac{1}{g^{2}}\left(\begin{array}{c}
2 g b_{x} \\
2 g b_{y} \\
2 u-\omega
\end{array}\right)+\sum_{j=0}^{n-1} \frac{\mathbf{s}_{j}}{u-\epsilon_{j}}
$$

also satisfies Eqs. (3.1). One can check using only Eq. (3.1) that the square of this vector commutes with itself at any two values, $v$ and $w$, of the auxiliary parameter, i.e.

$$
\left\{\mathbf{L}^{2}(v), \mathbf{L}^{2}(w)\right\}=0
$$

The function $\mathbf{L}^{2}(u)$ acts as a generating function for the model (2.3) and its integrals of motion. Indeed, evaluating $\mathbf{L}^{2}(u)$ from Eq. (3.2), we obtain

$$
\mathbf{L}^{2}(u)=\frac{(2 u-\omega)^{2}}{g^{4}}+\frac{4 H_{n}}{\omega g^{2}}+\sum_{j=0}^{n-1}\left[\frac{2 H_{j}}{g^{2}\left(u-\epsilon_{j}\right)}+\frac{\mathbf{s}_{j}^{2}}{\left(u-\epsilon_{j}\right)^{2}}\right] .
$$

where

$$
\begin{gathered}
H_{j}=\left(2 \epsilon_{j}-\omega\right) s_{j}^{z}+g\left(\bar{b} s_{j}^{-}+b s_{j}^{+}\right)+g^{2} \sum_{k \neq j} \frac{\mathbf{s}_{j} \cdot \mathbf{s}_{k}}{\epsilon_{j}-\epsilon_{k}}, \\
H_{n}=\omega\left(\bar{b} b+J_{z}\right) \quad \mathbf{J}=\sum_{j=0}^{n-1} \mathbf{s}_{j} .
\end{gathered}
$$

Hamiltonians $H_{j}$ defined by Eqs. (3.533.6) Poisson-commute with each other

$$
\left\{H_{j}, H_{k}\right\}=0 \quad \text { for all } j \text { and } k \text {. }
$$


This follows directly from the fact that Eq. (3.3) holds for any $v$ and $w$. On the other hand, it is straightforward to verify that the spin-oscillator Hamiltonian (2.3) is a linear combination of $H_{j}$

$$
H=\sum_{j=0}^{n} H_{j} .
$$

Since $H_{j}$ Poisson-commute with each other, the Hamiltonian (2.3) commutes with all $n+1$ Hamiltonians $H_{j}$. Therefore, $H_{j}$ given by Eqs. (3.53.6) are integrals of motion for the classical spin-oscillator model, i.e. this model is integrable.

The above construction based on Eqs. (3.1) and (3.2) constitutes the so-called Lax vector representation for the spin-oscillator model (2.3). Its main advantages are that it provides a powerful tool to study the dynamics and can also be extended to quantum models. In particular, note that integrals $(3.5)$ and (3.6) can be trivially generalized to the quantum case

$$
\begin{aligned}
& \hat{H}_{j}=\left(2 \epsilon_{j}-\omega\right) \hat{K}_{j}^{z}+g\left(\hat{b}^{\dagger} \hat{K}_{j}^{-}+\hat{b} \hat{K}_{j}^{+}\right)+g^{2} \sum_{j \neq k} \frac{\hat{\mathbf{K}}_{j} \hat{\mathbf{K}}_{k}}{\epsilon_{j}-\epsilon_{k}} \\
& \hat{H}_{n}=\omega\left(\hat{b}^{\dagger} \hat{b}+\hat{T}_{z}\right) \quad \hat{\mathbf{T}}=\sum_{q=0}^{n-1} \hat{\mathbf{K}}_{q} .
\end{aligned}
$$

Operators $\hat{H}_{j}$ pairwise commute with each other and the spin-oscillator Hamiltonian (1.3), which is their linear combination as in Eq. (3.7).

The same construction with classical dynamics in the mean-field approximation and Lax vector for resulting classical models applies to a number of other models. In particular, a closely related model is the BCS model

$$
\hat{H}_{B C S}=\sum_{j, \sigma} \epsilon_{j} \hat{c}_{j \sigma}^{\dagger} \hat{c}_{j \sigma}-g \sum_{j, q} \hat{c}_{j \uparrow}^{\dagger} \hat{c}_{j \downarrow}^{\dagger} \hat{c}_{q \downarrow} \hat{c}_{q \uparrow}
$$

Notations here are the same as in Eq. (1.1). In terms of Anderson's spins (see above Eq. (1.3)), the Hamiltonian reads

$$
\hat{H}_{B C S}=\sum_{j=0}^{n} 2 \epsilon_{j} \hat{K}_{j}^{z}-g \sum_{j, q} \hat{K}_{j}^{+} \hat{K}_{q}^{-} .
$$

The usual BCS mean-field is equivalent to the procedure (Eqs. (2.1) and (2.2)) we used to derive the classical Hamiltonian (2.3), only now the role of $\hat{b}$ is played by $\sum_{j} \hat{K}_{j}^{-}$(see e.g. Refs. 6, 9). The mean-field dynamics is described by the following classical Hamiltonian:

$$
H_{B C S}=\sum_{j=0}^{n} 2 \epsilon_{j} s_{j}^{z}-g \sum_{j, q} s_{j}^{+} s_{q}^{-}
$$


with the Lax vector

$$
\mathbf{L}_{B C S}(u)=-\frac{\hat{\mathbf{z}}}{g}+\sum_{j} \frac{\mathbf{s}_{j}}{u-\epsilon_{j}},
$$

where $\hat{\mathbf{z}}$ is a unit vector along the $z$-axis. The Lax vector $\mathbf{L}_{B C S}(u)$ has the same properties (3.1) and (3.3) as before and generates integrals of motion for the BCS model.

\section{SEPARATION OF VARIABLES}

In this section we perform a transformation to a new set of variables, which facilitates the solution of the equations of motion (2.2). The choice of new variables naturally follows from the Lax vector construction of the previous section. The new variables are canonical and also separate the Hamilton-Jacobi equations for the classical Hamiltonian (2.3) in the usual sense[36, 41].

We define $n$ variables $u_{j}$ as zeros of

$$
L_{-}(u)=L_{x}(u)-i L_{y}(u)=\frac{2 b}{g}+\sum_{j=0}^{n-1} \frac{s_{j}^{-}}{u-\epsilon_{j}} .
$$

Note that the nominator of this expression is a polynomial of degree $n$ and therefore there

are $n$ roots $u_{j}$ with $j=0, \ldots, n-1$. The coefficients of this polynomial are functions of the dynamical variables $s_{j}^{-}$and $b$. Accordingly, its roots are also functions of $s_{j}^{-}$and $b$ and therefore define a new set of dynamical variables.

Variables $u_{j}$ play a role of canonical coordinates for the classical Hamiltonian (2.3). The corresponding momenta are defined as $v_{j}=L_{z}\left(u_{j}\right)$. Thus, we have

$$
L_{-}\left(u_{j}\right)=0, \quad v_{j}=L_{z}\left(u_{j}\right), \quad j=0, \ldots, n-1
$$

Because our system has $2(n+1)$ degrees of freedom, we need two additional variables $u_{n}$ and $v_{n}$, which can be introduced as

$$
u_{n}=b, \quad v_{n}=\frac{H_{n}}{\omega b}=\bar{b}+\frac{J_{z}}{b}
$$

where $H_{n}$ is given by Eq. (3.6) .

Separation variables $\left(u_{j}, v_{j}\right)$ are canonical, i.e.

$$
\left\{u_{j}, u_{k}\right\}=0 \quad\left\{v_{j}, v_{k}\right\}=0 \quad\left\{v_{j}, u_{k}\right\}=-i \delta_{j k} \quad j, k=0, \ldots, n
$$


The first relation in Eq. (4.4) follows from the fact that by Eqs. (4.2) and (4.3) variables $u_{j}$ depend only on mutually Poisson-commuting variables $s_{k}^{-}$and $b$. The second relation follows from $\left\{L_{z}(v), L_{z}(w)\right\}=\left\{L_{z}(v), b\right\}=0$. To derive Poisson brackets between $v_{j}$ and $u_{k}$ for $j, k=0, \ldots, n-1$; we use the following equation obtained from Eq. (3.1)

$$
\left\{L_{z}(v), L_{-}(w)\right\}=\frac{i}{v-w}\left(L_{-}(v)-L_{-}(w)\right)
$$

Evaluating this expression at $v=u_{j}$ and $w=u_{k}$, we obtain

$$
\left\{L_{z}\left(u_{j}\right), L_{-}(w)\right\}_{w=u_{k}}=\lim _{w \rightarrow u_{k}} \frac{-i}{u_{j}-w} L_{-}(w)=i L_{-}^{\prime}\left(u_{k}\right) \delta_{j k} .
$$

where $L^{\prime}(u)=\partial L / \partial u$. On the other hand,

$$
\left\{L_{z}\left(u_{j}\right), L_{-}(w)\right\}_{w=u_{k}}=\left\{L_{z}\left(u_{j}\right), L_{-}\left(u_{k}\right)\right\}-L_{-}^{\prime}\left(u_{k}\right)\left\{L_{z}\left(u_{j}\right), u_{k}\right\}=-L_{-}^{\prime}\left(u_{k}\right)\left\{L_{z}\left(u_{j}\right), u_{k}\right\}
$$

where we used $L_{-}\left(u_{k}\right)=0$. Comparing Eqs. (4.6) and (4.7), we obtain the last relation in Eq. (4.4) for $j, k=0, \ldots, n-1$.

The original dynamical variables $\mathbf{s}_{j}=\left\langle\hat{\mathbf{K}}_{j}(t)\right\rangle$ and $b=\langle\hat{b}(t)\rangle$ that we are interested in, can be explicitly expressed in terms of $u_{j}$ (the inverse map). Indeed, note that $L_{-}(u)$ has its zeros at $u=u_{j}$ and poles at $u=\epsilon_{j}$. Therefore, using Eqs. (4.2) and (4.1), we can write it as

$$
L_{-}(u)=\frac{2 b}{g}+\sum_{j=0}^{n-1} \frac{s_{j}^{-}}{u-\epsilon_{j}}=\frac{2 b}{g} \frac{\prod_{k}\left(u-u_{k}\right)}{\prod_{j}\left(u-\epsilon_{j}\right)},
$$

where we have also used $L_{-}(u)=2 b / g+O(1 / u)$ for large $u$. Equating residues at $u=\epsilon_{j}$ and $u=\infty$, we obtain

$$
\begin{gathered}
s_{j}^{-}=\frac{2 b}{g} \frac{\prod_{k}\left(\epsilon_{j}-u_{k}\right)}{\prod_{l \neq j}\left(\epsilon_{j}-\epsilon_{l}\right)}, \\
J_{-}=\sum_{j} s_{j}^{-}=\frac{2 b}{g} \sum_{j=0}^{n-1}\left(\epsilon_{j}-u_{j}\right) .
\end{gathered}
$$

Similarly, using

$$
L_{z}\left(u_{j}\right)=v_{j} \quad L_{z}(u)=\frac{2 u-\omega}{g^{2}}+O(1 / u)
$$

one can express $L_{z}(u)$ in terms of $u_{j}$. The $z$-components of classical spins, $s_{j}^{z}$, are residues of $L_{z}(u)$ at $u=\epsilon_{j}($ see Eq. (3.2) $)$

$$
s_{j}^{z}=s_{j}^{-}\left[\frac{\epsilon_{j}-\omega / 2+\sum_{k}\left(u_{k}-\epsilon_{k}\right)}{b g}+\sum_{k} \frac{v_{k}}{\left(\epsilon_{j}-u_{k}\right) L_{-}^{\prime}\left(u_{k}\right)}\right] .
$$


It also follows from Eq. (4.2) that $v_{j}^{2}=\mathbf{L}^{2}\left(u_{j}\right)$. This allows us to express variables $v_{j}$ through $u_{j}$ and the integrals of motion $H_{j}$

$$
\begin{gathered}
v_{j}^{2}=\frac{\left(2 u_{j}-\omega\right)^{2}}{g^{4}}+\frac{4 H_{n}}{\omega g^{2}}+\sum_{k=0}^{n-1}\left[\frac{2 H_{k}}{g^{2}\left(u_{j}-\epsilon_{k}\right)} \frac{\mathbf{s}_{k}^{2}}{\left(u_{j}-\epsilon_{k}\right)^{2}}\right] \quad j=0, \ldots, n-1 \\
v_{n}=\frac{H_{n}}{\omega u_{n}}=\frac{H_{n}}{\omega b} .
\end{gathered}
$$

Thus, to determine the evolution of $\mathbf{s}_{j}(t)=\left\langle\hat{\mathbf{K}}_{j}(t)\right\rangle$ and $b(t)=\langle\hat{b}(t)\rangle$ we only need to derive and solve the equations of motion for $u_{j}(t)$.

\section{EQUATIONS OF MOTION FOR SEPARATION VARIABLES}

In order to derive the equations of motion for separation variables $u_{j}$, we first determine the brackets $u_{l, k}=\left\{H_{k}, u_{l}\right\}$ for $l, k=0, \ldots, n-1$ using Eqs. (4.12) and (4.4). This is done by taking Poisson brackets of both sides of Eq. (4.12) with $u_{l}$ and solving the resulting system of linear equations for $u_{l, k}$.

As soon as $u_{l, k}$ are found in terms of $u_{j}$ and $v_{j}$, we can use the expression (3.7) for the Hamiltonian in terms of $H_{j}$ to determine $\dot{u}_{j}$

$$
\dot{u}_{j}=\left\{H, u_{j}\right\}=\sum_{k} u_{j, k}
$$

Taking Poisson brackets of both sides of Eqs. (4.12) and (4.13) with $u_{l}$, we obtain

$$
-i g^{2} v_{j} \delta_{j l}=\sum_{k=0}^{n-1} \frac{u_{l, k}}{u_{j}-\epsilon_{k}} \quad j, l=0, \ldots n-1 .
$$

In order to determine $u_{n, k}$ and $u_{l, k}$ for $l, k=0, \ldots, n-1$, we need to invert the matrix

$$
A_{j k}=\frac{1}{u_{j}-\epsilon_{k}}
$$

which is called the Cauchy matrix.

This can be done with the help of the following identity coming from partial fraction decomposition of the LHS:

$$
\frac{\prod_{l=0}^{n-1}\left(u-u_{l}\right)}{\left(u-u_{p}\right) \prod_{l=0}^{n-1}\left(u-\epsilon_{l}\right)}=\sum_{j=0}^{n-1} \frac{1}{u-\epsilon_{j}} \frac{\prod_{l \neq p}\left(\epsilon_{j}-u_{l}\right)}{\prod_{l \neq j}\left(\epsilon_{j}-\epsilon_{l}\right)} .
$$


To verify this identity note that both sides have the same poles and that the residues at these poles coincide. Substituting $u=u_{q}$ in Eq. (5.4), one derives

$$
\delta_{q p}=\sum_{j=0}^{n-1} \frac{1}{u_{q}-\epsilon_{j}} \frac{\prod_{l=1}^{n}\left(u_{p}-\epsilon_{l}\right)}{\prod_{l \neq j}\left(\epsilon_{j}-\epsilon_{l}\right)} \prod_{l \neq p} \frac{\epsilon_{j}-u_{l}}{u_{p}-u_{l}} .
$$

Hence

$$
\left(A^{-1}\right)_{j k}=\frac{\prod_{l=1}^{n}\left(u_{k}-\epsilon_{l}\right)}{\prod_{l \neq j}\left(\epsilon_{j}-\epsilon_{l}\right)} \prod_{l \neq k} \frac{\epsilon_{j}-u_{l}}{u_{k}-u_{l}} .
$$

It follows from Eqs. (5.2) and (4.9) that

$$
u_{j, k}=-i g^{2} v_{j} \frac{\prod_{l=0}^{n-1}\left(u_{j}-\epsilon_{l}\right)}{\prod_{l \neq j}\left(u_{j}-u_{l}\right)} \frac{g s_{k}^{-}}{2 b\left(\epsilon_{k}-u_{j}\right)} .
$$

Finally, noticing that $0=L_{-}\left(u_{j}\right)=2 b / g+\sum_{k} s_{k}^{-} /\left(u_{j}-\epsilon_{k}\right)$ and using Eqs. (5.1) and (4.12), we derive

$$
\begin{aligned}
& \dot{u}_{j}=-\frac{2 i \sqrt{Q_{2 n+2}\left(u_{j}\right)}}{\prod_{m \neq j}\left(u_{j}-u_{m}\right)} \quad j=0, \ldots, n-1 \\
& \dot{b}=-2 i b\left(\frac{\omega}{2}+\sum_{j=0}^{n-1} \epsilon_{j}-\sum_{k=0}^{n-1} u_{k}\right),
\end{aligned}
$$

where the spectral polynomial $Q_{2 n+2}(u)$ is defined as

$$
Q_{2 n+2}(u)=g^{4} \mathbf{L}^{2}(u) \prod_{j=0}^{n-1}\left(u-\epsilon_{j}\right)^{2} .
$$

By Eq. (3.4), the coefficients of $Q_{2 n+2}(u)$ depend only on the integrals of motion $H_{j}$ and parameters $\epsilon_{j}$ and $g$. The equation of motion for $u_{n}=b$ is obtained by substituting Eq. (4.10) into Eq. (2.2).

An almost identical derivation of equations of motion for separation variables can be performed for the mean-field BCS model (3.10) using its vector Lax representation (3.11) leading to the following equations of motion:

$$
\begin{aligned}
& \dot{u}_{j}=-\frac{2 i \sqrt{Q_{2 n+2}^{B C S}\left(u_{j}\right)}}{\prod_{m \neq j}\left(u_{j}-u_{m}\right)} \quad j=0, \ldots, n-1 \\
& \dot{J}_{-}=-2 i J_{-}\left(g J_{z}+\sum_{j=0}^{n} \epsilon_{j}-\sum_{k=0}^{n-1} u_{k}\right),
\end{aligned}
$$

where the spectral polynomial for the BCS model is defined as

$$
Q_{2 n+2}^{B C S}(u)=\frac{1}{g^{2}} \mathbf{L}_{B C S}^{2}(u) \prod_{j=0}^{n}\left(u-\epsilon_{j}\right)^{2} .
$$




\section{THE SOLUTION}

Here we obtain the general solution for the mean-field dynamics of the fermion-oscillator (spin-oscillator) model (1.11.3) by mapping it onto the corresponding BCS model. The solution therefore can be read off from the known general solution of the mean-field BCS problem [9].

By comparing equations of motion (5.8) for the spin-oscillator model with those for BCS model (5.10), we observe that they coincide upon a replacement

$$
\sum_{j=0}^{n} \epsilon_{j}+g J_{z} \rightarrow \sum_{j=0}^{n-1} \epsilon_{j}+\frac{\omega}{2}, \quad J_{-} \rightarrow b, \quad Q_{2 n+2}^{B C S}(u) \rightarrow Q_{2 n+2}(u) .
$$

Thus, the mean-field dynamics of the spin-oscillator model (1.3) with $n$ spins and a bosonic field coincides with that of the BCS model (3.9) with $n+1$ spins. This allows us to immediately write down the solution for the time-dependent averages $\mathbf{s}_{j}(t)=\left\langle\hat{\mathbf{K}}_{j}(t)\right\rangle$ and $b(t)=\langle\hat{b}(t)\rangle$

$$
b(t)=\left[J_{-}\right]_{B C S}, \quad\left[\mathbf{s}_{j}(t)\right]_{\text {Dicke }}=\left[\mathbf{s}_{j}(t)\right]_{B C S} .
$$

The explicit form of $\left[J_{-}\right]_{B C S}$ and $\left[\mathbf{s}_{j}(t)\right]_{B C S}$ in terms of hyperelliptic functions can be found in Ref. 9 (see Eqs. $(3.22-3.24)$ of Ref. 9).

The dynamics of $\mathbf{s}_{j}(t)=\left\langle\hat{\mathbf{K}}_{j}(t)\right\rangle$ and $b(t)=\langle\hat{b}(t)\rangle$ is typical of a classical integrable system with $n+1$ degrees of freedom [36] (recall that $n$ is the number of non-degenerate single particle levels $\epsilon_{j}$ in the fermion-oscillator model (1.1)). The typical motion is quasi-periodic with $n+1$ incommensurate periods. For example, a Fourier transform of $b(t)=\langle\hat{b}(t)\rangle$ shows $n+1$ basic frequencies. The system uniformly (ergodically) explores the invariant torus - the $n+1$-dimensional portion of the $2 n+2$-dimensional phase space allowed by the integrals of motion (3.5) and (3.6), returning arbitrarily close to the initial point at irregular intervals.

We found that in the thermodynamic limit $n \rightarrow \infty$ the solution simplifies for most physical initial conditions. The return time diverges in this limit. The dynamics of $b(t)$ is particularly simple - it decays, typically as a power-law, to a steady state where $|b(t)|$ is either constant or is characterized by a few independent frequencies [31]. However, the motion of the spin system still contains a continuum of frequencies. The final steady state of $|b(t)|$ depends on the initial conditions. 


\section{FEW SPIN SOLUTIONS}

The evolution described in the previous section occurs for most initial conditions and is stable against small perturbations of the Hamiltonian by the KAM theorem even if these perturbations destroy the integrability. However, there always exists a set of points of measure zero in the phase space, where the motion is characterized by only a few incommensurate frequencies, while the stability is not guaranteed [36]. Below we consider a family of such solutions, which we call $m$-spin solutions with $m<n$. The reason is that in these cases the dynamics of $n$ spins and the bosonic field in Hamiltonian (1.3) degenerates to that of $m<n$ spins and the bosonic field.

Few spin solutions are constructed by choosing integrals of motion $H_{j}$ (i.e. the initial conditions) so that $2(n-m)$ roots of the spectral polynomial $Q_{2 n+2}(u)$ defined in Eq. (3.4) become double degenerate. Suppose $u=E_{0}$ is a double root [43] of $Q_{2 n+2}(u)$ and note that, since $Q_{2 n+2}\left(E_{0}\right)=\mathbf{L}^{2}\left(E_{0}\right)=0$, setting e.g. $u_{n-1}(t)=E_{0}$ solves the equation of motion (5.8) for the variable $u_{n-1}$. Therefore, we can "freeze" one of the separation variables in this root. Then $L_{-}\left(E_{0}\right)=0$, and it follows from $L_{z}^{2}(u)+L_{-}(u) L_{+}(u)=\mathbf{L}^{2}(u)$ that $L_{z}\left(E_{0}\right)=0$. Thus, one can factor out $\left(u-E_{0}\right)$ from all components of the Lax vector and show that it is proportional to the Lax vector of the spin-oscillator model with $n-1$ spins. Similarly, if there are 2 pairs of double degenerate roots, the number of spins reduces to $n-2$ etc. This procedure is followed in detail in Ref. 9 using a different method.

First, let us consider the general case when the number of spins effectively reduces from $n$ to $m<n$. We have

$$
\mathbf{L}(u)=\left(1+\sum_{j} \frac{d_{j}}{u-\epsilon_{j}}\right) \mathbf{L}_{\mathbf{t}}(u),
$$

where $d_{j}$ are time-independent constants and $\mathbf{L}_{\mathbf{t}}(u)$ is the Lax vector of the $m$-spin problem

$$
\mathbf{L}_{\mathbf{t}}(u)=\frac{1}{g^{2}}\left(\begin{array}{c}
2 g b_{x} \\
2 g b_{y} \\
2 u-\omega^{\prime}
\end{array}\right)+\sum_{k=0}^{m-1} \frac{\mathbf{t}_{k}}{u-\mu_{k}} .
$$

The $m$-spin system has its own $m$ arbitrary "energy levels" $\mu_{k}$. Its dynamics is governed by the spin-oscillator Hamiltonian (2.3) for $m$ spins with new parameters replacing $\epsilon_{j}$ and $\omega$.

$$
H_{m}=\sum_{k=0}^{m-1} 2 \mu_{k} t_{k}^{z}+\omega^{\prime} \bar{b} b+g \sum_{k=0}^{m-1}\left(\bar{b} t_{k}^{-}+b t_{k}^{+}\right) \text {. }
$$


Matching the residues at poles at $u=\epsilon_{j}$ on both sides of Eq. (7.1), we express the original spins $\mathbf{s}_{j}$ in terms of collective spins $\mathbf{t}_{k}$

$$
\mathbf{s}_{j}=d_{j} \mathbf{L}_{\mathbf{t}}\left(\epsilon_{j}\right) .
$$

Constants $d_{j}$ are determined from the above equation using $\mathbf{s}_{j}^{2}=1 / 4$. We have

$$
d_{j}=\frac{\left|\mathbf{s}_{j}\right| e_{j}}{\sqrt{\mathbf{L}_{\mathbf{t}}^{2}\left(\epsilon_{j}\right)}}=\frac{e_{j}}{2 \sqrt{\mathbf{L}_{\mathbf{t}}^{2}\left(\epsilon_{j}\right)}} \quad e_{j}= \pm 1 .
$$

Finally we have to match the residues at $u=\mu_{k}$ and the $u \rightarrow \infty$ asymptotic. This leads to the following $m+1$ equations

$$
\begin{aligned}
& 1+\sum_{j=0}^{n-1} \frac{d_{j}}{\mu_{k}-\epsilon_{j}}=0 \quad k=0, \ldots, m-1 \\
& \omega=\omega^{\prime}-2 \sum_{j=0}^{n-1} d_{j} .
\end{aligned}
$$

Equations (7.6) constrain the lengths of new spins $\mathbf{t}_{k}$, i.e. the coefficients of the spectral polynomial $Q_{2 m+2}(u)=g^{4} \mathbf{L}_{\mathbf{t}}^{2}(u) \prod_{k=0}^{m-1}\left(u-\mu_{k}\right)^{2}$ of the $m$-spin system (see Eq. (3.4) ). Indeed, using Eq. (7.5), one can cast the constraints Eq (7.6) into the form

$$
\begin{gathered}
\sum_{j=0}^{n-1} \frac{e_{j} \epsilon_{j}^{l-1}}{\sqrt{Q_{2 m+2}\left(\epsilon_{j}\right)}}=\frac{2}{g^{2}} \delta_{l m} \quad l=1, \ldots, m \\
\omega^{\prime}=\omega+2 \sum_{k=0}^{m-1} \mu_{k}-\sum_{j=0}^{n-1} \frac{g^{2} e_{j} \epsilon_{j}^{m}}{\sqrt{Q_{2 m+2}\left(\epsilon_{j}\right)}} .
\end{gathered}
$$

To obtain $m$-spin solutions explicitly, one has to choose parameters $\mu_{k}$, resolve the $m+1$ constraints (7.7) and (7.8) for the lengths of spins $\mathbf{t}_{k}$ and frequency $\omega^{\prime}$, and solve for the dynamics of the $m$-spin Hamiltonian (7.3). The dynamics can be obtained from the general solution (6.2) by replacing $n \rightarrow m$ and the set of $\left\{\epsilon_{j}\right\}$ with $\left\{\mu_{k}\right\}$.

Let us illustrate the construction of $m$-spin solutions by considering the cases $m=0$ and $m=1$ in more detail.

$\mathbf{m}=\mathbf{0}$. The 0 -spin solutions correspond to the mean-field eigenstates of quantum Hamiltonians (1.1) and (1.3). They are equilibrium states for the classical Hamiltonian (2.3).

For $m=0$ the dynamics is governed by the Hamiltonian $H_{0}=\omega^{\prime} \bar{b} b$ with an obvious solution $b=b_{0} e^{-i\left(\omega^{\prime} t+\phi\right)}$, where $b_{0}=|b(t)|=$ const. Further, using Eqs. (7.2), (17.4), and 
(17.5), we obtain

$$
\begin{gathered}
\mathbf{L}_{\mathbf{t}}^{2}(u)=\frac{1}{g^{4}}\left(4 g^{2} b_{0}^{2}+\left(2 u-\omega^{\prime}\right)^{2}\right), \\
\left\langle\hat{c}_{j \downarrow} \hat{c}_{j \uparrow}\right\rangle=s_{j}^{-}=\frac{e_{j} g b}{\sqrt{4 g^{2} b_{0}^{2}+\left(2 \epsilon_{j}-\omega^{\prime}\right)^{2}}}, \quad\left\langle\hat{n}_{j}\right\rangle-1=2 s_{j}^{z}=\frac{2 e_{j}\left(2 \epsilon_{j}-\omega^{\prime}\right)}{\sqrt{4 g^{2} b_{0}^{2}+\left(2 \epsilon_{j}-\omega^{\prime}\right)^{2}}} .
\end{gathered}
$$

The frequency $\omega^{\prime}$ and the gap $\Delta_{0}=g b_{0}$ have to satisfy a single constraint (77.8) that now reads

$$
\omega^{\prime}=\omega-\sum_{j=0}^{n-1} \frac{g^{2} e_{j}}{\sqrt{4 g^{2} b_{0}^{2}+\left(2 \epsilon_{j}-\omega^{\prime}\right)^{2}}} .
$$

We see from Eq. (7.10), that all spins rotate uniformly around the $z$-axis with a frequency $\omega^{\prime}$. This rotation can be eliminated, i.e. $\omega^{\prime}$ can be set to zero, by an appropriate choice of the chemical potential. Then, Eq. (7.11) is the analog of the BCS gap equation. The configuration of spins (7.10) corresponds to the product BCS wavefunction. The latter can be straightforwardly reconstructed from the knowledge of $\mathbf{s}_{j}=\left\langle\hat{\mathbf{K}}_{j}\right\rangle$ as fixing the average of spin-1/2 uniquely fixes its quantum state. The choice of signs $e_{j}=+1$ for all $j$ corresponds to the ground state, while choosing one of the signs to be -1 corresponds to an excited state - pair excitation of the fermionic condensate.

There is also an important class of 0 -spin solutions that is obtained by setting $b_{0}=0$. We see from Eq. (7.10) that in this case all spins are along the $z$-axis, $s_{j}^{z}=e_{j} / 2= \pm 1 / 2$. The constraint (7.11) is now irrelevant, because all $x y$-components vanish. The choice of signs $e_{j}=\operatorname{sign} \epsilon_{j}$, where $\epsilon_{j}$ are counted from the Fermi level, yields the Fermi ground state. Other choices correspond to the excitations of the Fermi gas. All these states are stationary with respect to the mean-field dynamics. For a finite number of pairs, they are non-stationary with respect to the quantum Hamiltonian (1.1). Thus, their initial evolution is entirely governed by quantum corrections (cf. Refs. 10, 11, 34).

$\mathbf{m}=\mathbf{1}$. In this case the dynamics reduces to that of a single collective spin $\mathbf{t}$ coupled to an oscillator, i.e. it is governed by the classical counterpart of the Dicke model (1.4)

$$
H_{1}=2 \mu t_{z}+\omega^{\prime} \bar{b} b+g\left(\bar{b} t_{-}+b t_{+}\right) .
$$

Using Eq. (7.10), one can express original spins in terms of the collective spin and the bosonic field

$$
s_{j}^{-}=\frac{e_{j}\left(\epsilon_{j}-\mu\right) b+g e_{j} t_{-}}{2 g \sqrt{Q_{4}\left(\epsilon_{j}\right)}} \quad s_{j}^{z}=\frac{e_{j}\left(2 \epsilon_{j}-\omega^{\prime}\right)\left(\epsilon_{j}-\mu\right)+g^{2} t_{z}}{2 g^{2} \sqrt{Q_{4}\left(\epsilon_{j}\right)}} .
$$


To complete the construction of 1-spin solutions, we need to choose a positively defined polynomial $Q_{4}(u)$ so that it satisfies two constraints (7.7) (17.8) and solve for the dynamics of the classical Dicke model (7.12). In this case there is only one non-stationary separation variable $u_{0}$ and equations of motion (5.8) take the following form:

$$
\begin{aligned}
& \dot{u}_{0}^{2}+4 Q_{4}\left(u_{0}\right)=0, \\
& \dot{b}=-i b\left(\omega+2 \mu-2 u_{0}\right) .
\end{aligned}
$$

These equations can be solved in terms of elliptic functions. This is not surprising, since the model (7.12) is, in fact, equivalent to a spherical pendulum and its solution has already been obtained in Refs. [32, 34].

The 1-spin solutions were originally obtained in Refs. [12, 13] where they were used to describe the evolution beginning from a state infinitesimally close to the normal ground state.

\section{CONCLUSION}

In this paper we solved for the mean-field dynamics of the fermion-oscillator model (1.1). In the mean-field approximation, the problem reduces to a classical Hamiltonian model. We derived integrals of motion for both the classical (2.3) and the quantum (1.1) models and showed that the dynamics of the fermion-oscillator model (1.1) maps onto that of the BCS model. This was used to derive an explicit general solution for the mean-field dynamics of the fermion-oscillator model with an arbitrary finite number $n$ of degrees of freedom.

The typical dynamics is quasi-periodic with $n$ incommensurate frequencies. The system ergodically explores the part of the phase-space allowed by integrals of motion, returning arbitrarily close to its initial state at irregular time intervals. We have also constructed a class of particular, few-spin, solutions, for which the dynamics reduces to that of $m$ collective spins governed by the same classical model. The case $m=0$ corresponds to the mean-field eigenstates of the fermion-oscillator Hamiltonian (1.1).

An interesting problem is to obtain and describe the thermodynamic limit, $n \rightarrow \infty$, of the solution. For most physical initial conditions the dynamics considerably simplifies in

this limit [31]. In particular, we found that $\langle\hat{b}(t)\rangle$ decays, typically as a power-law, to a steady state where $|\langle\hat{b}(t)\rangle|$ is either a constant (cf. Refs. 2, 3, 7, 11) or oscillates with few 
independent frequencies (cf. Fig. 2 in Refs. 4, 66). The motion of the spin system still contains a continuum of frequencies. The steady state of $|\langle\hat{b}(t)\rangle|$ depends on the initial conditions.

It is also interesting to study quantum and finite temperature effects. Quantum corrections to the mean-field dynamics can become important when the bosonic mode is weakly populated [34]. For example, normal states where $\left\langle\hat{b}^{\dagger} \hat{b}\right\rangle=\left\langle\hat{c}_{j \downarrow} \hat{c}_{j \uparrow}\right\rangle=0$ are stationary in the mean-field approximation (2.2), but are not stationary with respect to quantum dynamics (2.1).

[1] P. W. Anderson: Phys. Rev. 112, 1900 (1958).

[2] V. P. Galaiko: Sov. Phys. JETP 34, 203 (1972).

[3] A. F. Volkov and Sh. M. Kogan: Sov. Phys. JETP 38, 1018 (1974).

[4] Yu. M. Gal'perin, V. I. Kozub, and B. Z. Spivak: Sov. Phys. JETP 54, 1126 (1981).

[5] V. S. Shumeiko: Dynamics of electronic system with off-diagonal order parameter and nonlinear resonant phenomena in superconductors, Doctoral Thesis, Kharkov, 1990.

[6] R. A. Barankov, L. S. Levitov, and B. Z. Spivak: Phys. Rev. Lett. 93, 160401 (2004).

[7] M. H. Szymanska, B. D. Simons, and K. Burnett: Phys. Rev. Lett. 94 , 170402 (2005).

[8] M.H.S. Amin, E.V. Bezuglyi, A.S. Kijko, A.N. Omelyanchouk: Low Temp. Phys. 30, 661 (2004).

[9] E. A. Yuzbashyan, B. L. Altshuler, V. B. Kuznetsov and V. Z. Enolskii: cond-mat/0407501.

[10] E. A. Yuzbashyan, B. L. Altshuler, V. B. Kuznetsov and V. Z. Enolskii: cond-mat/0505493.

[11] G. L. Warner and A. J. Leggett, Phys. Rev. B 71134514 (2005).

[12] A. V. Andreev, V. Gurarie, and L. Radzihovsky: Phys. Rev. Lett. 93, 130402 (2004).

[13] R. A. Barankov and L. S. Levitov: Phys. Rev. Lett. 93, 130403 (2004).

[14] N. V. Prokof'ev and P. C. E. Stamp: J. Phys.: Cond. Mat. 5, L663 (1993); In: Quantum Tunneling of Magnetization (ed. L. Gunther and B. Barbara), Kluwer (1995).

[15] A. V. Khaetskii, D. Loss, and L. Glazman: Phys. Rev. Lett. 88, 186802 (2002)

[16] J. Schliemann, A. Khaetskii, and D. Loss: J. Phys., Condens. Matter 15, R1809 (2003).

[17] S. I. Erlingsson and Yu. V. Nazarov: cond-mat/0405318.

[18] W. A. Coish and D. Loss: Phys. Rev. B 70, 195340 (2004).

[19] A. Melikidze, V. V. Dobrovitski, H. A. De Raedt, M. I. Katsnelson, and B. N. Harmon: Phys. 
Rev. B, 70, 14435 (2004).

[20] J. M. Taylor, A. Imamoglu, and M. D. Lukin: Phys. Rev. Lett. 91, 246802 (2003).

[21] M. Gaudin: Note CEA 1559, 1 (1972); J. Phys. (Paris) 37, 1087 (1976); La fonction d'onde de Bethe, Masson, Paris (1983).

[22] E. K. Sklyanin: J. Sov. Math. 47, 2473 (1989); Progr. Theoret. Phys. Suppl. 118, 35 (1995).

[23] V. B. Kuznetsov: J. Math. Phys. 33, 3240, (1992).

[24] C. A. Regal, M. Greiner, and D. S. Jin: Phys. Rev. Lett. 92, 040403 (2004).

[25] M. W. Zwierlein et. al.: Phys. Rev. Lett. 92, 120403 (2004).

[26] J. Kinast et. al.: Phys. Rev. Lett. 92, 150402 (2004).

[27] M. W. Zwierlein et. al.: Phys. Rev. Lett. 94, 180401 (2005).

[28] T. Loftus et al., Phys. Rev. Lett. 88, 173201 (2002); K. Dieckmann et al., Phys. Rev. Lett. 89, 203201 (2002); S. Jochim et al., Phys. Rev. Lett. 89, 273202 (2002).

[29] H. Feshbach, Ann. Phys. 5, 357 (1958); Theoretical Nuclear Physics, Wiley, New York, (1992).

[30] P. W. Anderson: J. Phys. Chem. Solids 11, 26 (1959).

[31] E. A. Yuzbashyan, B. L. Altshuler, and O. Tsyplyatev: unpublished.

[32] R. H. Dicke: Phys. Rev. 93, 99 (1954)

[33] M. Tavis and F. W. Cummings: Phys. Rev. 170, 379 (1968).

[34] R. Bonifacio and G. Preparata: Phys. Rev. A 2, 336 (1970).

[35] B. Jurco: J. Math. Phys. 30, 1739 (1989)

[36] V. I. Arnold: Mathematical Methods of Classical Mechanics, Springer-Verlag, New York, (1978); M. Tabor, Chaos and Integrability in Nonlinear Dynamics, Wiley, New York, (1989).

[37] I. V. Komarov, unpublished.

[38] E. D. Belokolos, A. I. Bobenko, V. Z. Enol'skii, A. R. Its, and V. B. Matveev: Algebrogeometric approach to nonlinear-integrable equations, Springer-Verlag, Berlin (1994).

[39] D. Mumford: Tata Lectures on Theta, vols 1-2. Birkhauser (1983, 1984); H.F. Baker: An introduction to the theory of Multiply Periodic Functions, Cambridge University Press (1907).

[40] The coordinate, $x$, and the momentum, $p$ of the oscillator are related to the fields $b$ and $\bar{b}$ as $x=\sqrt{2} b_{x}=(b+\bar{b}) / \sqrt{2}$ and $p=\sqrt{2} b_{y}=(\bar{b}-b) /(\sqrt{2} i)$. Then, we have the usual coordinatemomentum (canonical) brackets $\{x, p\}=1$, while the second term in the Hamiltonian (2.3) reads $\bar{b} b=p^{2} / 2+x^{2} / 2$.

[41] L. D. Landau and E. M. Lifshitz: Mechanics, 3rd edition, Butterworth-Heinemann, Burlington 
(1976).

[42] V.M. Buchstaber, V. Z. Enolskii, and D. V. Leykin: Hyperelliptic Kleinian functions and applications, in Solitons, Geometry and Topology: On the Crossroad, (eds. V.M. Buchstaber and S.P. Novikov), AMS Translations Series 2, Vol. 179, American Mathematical Society (1997) 1-34.

[43] Note that if the root $E_{0}$ is real, it has to be degenerate, since according to Eq. (5.9) $Q_{2 n+2}(u) \geq$ 0 for real $u$. In this case $\mathbf{L}^{2}\left(E_{0}\right)=0$ implies $L_{z}\left(E_{0}\right)=L_{-}\left(E_{0}\right)$ and, since zeros of $L_{-}(u)$ are separation variables, one of them has to be frozen at $E_{0}$. 